\title{
Nanosized silver-palm pollen nanocomposite, green synthesis, characterization and antimicrobial activity
}

\begin{abstract}
Palm pollen (PP) has been widely used in nutrition, pharmaceutical and cosmetic industries. In the present study, we explored the potential of PP in the synthesis of a silver nanoparticle (Ag NP). PP was used as both reducing and stabilizing agent. The Ag/PP nanocomposite was examined by field emission electron microscopy, X-ray diffraction, Fourier transform infrared (FT-IR) spectroscopy, ultraviolet spectroscopy and zeta potential measurement. The biosynthesized NPs showed surface plasmon resonance centered at $425 \mathrm{~nm}$ with an average particle size measured to be $23 \mathrm{~nm}$ and a zeta potential of i $30.9 \mathrm{mV}$. Prominent FT-IR signals were obtained and ascribed to phenolic and carbohydrate compounds involved in the formation of the Ag NPs, and proteins which participated in stabilization of the Ag NPs. The biologically synthesized Ag NPs were found to be extremely effective against E. coli (13.8 \pm $0.25 \mathrm{~mm}$ ) with a minimum inhibitory concentration of $20 \mu \mathrm{g} / \mathrm{mL}$. Thus, such biosynthesized Ag NPs can be used in medicinal applications.
\end{abstract}

Keyword: Antibacterial; Green synthesis; Palm pollen; Porous scaffold; Silver nanoparticles 